\title{
Mit Licht aus der düsteren Stimmung
}

Fragestellung: Wirkt Lichttherapie allein oder in Kombination mit Fluoxetin versus Sham-Placebo-Therapie auch bei der nicht saisonalen Major Depression (MD)?

Hintergrund: Die MD ist mit erheblichen psychosozialen Belastungen für die Betroffenen und ihre Familien sowie mit einer erhöhten Mortalität assoziiert. Trotz vorhandener psychotherapeutischer und medikamentöser Behandlungsoptionen werden nur relativ geringe Remissionsraten (zirka 67\%) erzielt, sodass erheblicher Bedarf an weiteren Therapiemöglichkeiten besteht. Mithilfe der Lichttherapie können saisonale affektive Störungen wirksam behandelt werden. Aufgrund ihres korrigierenden Einflusses auf die zirkadiane Rhythmik, deren Störung auch in der Pathophysiologie der MD eine wesentliche Rolle spielt, könnte die Lichttherapie auch in dieser Indikation Bedeutung erlangen. Die Lichttherapie ist ein sicheres, gut verträgliches nicht medikamentöses Verfahren, das alleine oder in Kombination mit einer Medikation angewandt werden kann. Bisherige Studien und Metaanalysen zur Wirksamkeit bei der nicht saisonalen MD erbrachten widersprüchliche Befunde, die sicher auch durch deren heterogene Qualität mit zu erklären sind. Bei der vorliegenden Untersuchung handelt es sich um die erste randomisierte kontrollierte Studie

Lam RW, MD; Levitt AJ, Levitan RD et al. Efficacy of bright light treatment, fluoxetine, and the combination in patients with nonseasonal major depressive disorder. A randomized clinical trial. JAMA Psychiatry 2016; 73 : $56-63$ zur Lichttherapie bei MD.

Patienten und Methodik: In die randomisierte, doppelblinde, placebo- und shamkontrollierte achtwöchige Studie wurden 122 ambulante Patienten zwischen 19 und 60 Jahren mit mindestens mittelgradiger Depression eingeschlossen. Die bei Studieneinschluss mindestens seit zwei Jahren medikamentenfreien Patienten wurden randomisiert folgenden Bedingungen zugeteilt: 1) Lichtmonotherapie (flächige weiße 10.000 Lux LED-Lampe für $30 \mathrm{~min} / \mathrm{Tag}$ schnellstmöglich nach dem Aufwachen plus Placebo), 2) Fluoxetinmonotherapie (inaktiver Ionenstrahler kombiniert mit Fluoxetin $20 \mathrm{mg} / \mathrm{Tag}$ ), 3) Kombination aus Lichttherapie und Fluoxetin, 4) Placebo (inaktiver Ionenstrahler plus Placebo). Primärer Endpunkt war die Veränderung auf der Montgomery Åsberg Depression Rating Scale (MADRS). Sekundäre Endpunkte waren Response ( $\geq 50 \%$ MADRS-Score-Reduktion) und Remission (MADRS Score $\leq 10$ nach acht Wochen).

Ergebnisse: Von 122 randomisierten Patienten erhielten 32 Lichtmonotherapie, 31 Fluoxetinmonotherapie, 29 eine Kombinationstherapie und 30 Placebo. Die mittleren Veränderungen der MADRS-Scores betrugen 13,4 unter Lichtmonotherapie, 8,8 unter Fluoxetinmonotherapie, 16,9 unter Kombinationstherapie und 6,5 unter Placebo. Hinsichtlich der Veränderung im MADRS-Score waren die Kombinationsbehandlung und die Lichtmonotherapie Placebo signifikant überlegen. Die Fluoxetinmonotherapie war Placebo jedoch nicht überlegen. Nach acht Wochen erzielten in der Placebo-, Fluoxetin-, Licht- und Kombinationsbedingung jeweils 10 (33,3\%), 9 (29,0\%), 16 (50,0\%) und $22(75,9 \%)$ Patienten eine Therapieresponse und 9 (30,0\%), $6(19,4 \%), 14(43,8 \%)$ und 17 (58,6\%) eine Remission. Hinsichtlich der MADRS-Response und -Remission war die Kombinations- der Placebobehandlung überlegen.

Schlussfolgerungen: Lichttherapie allein und in Kombination mit Fluoxetin erwies sich in der Therapie der nicht saisonalen, mittelgradigen MD als wirksam und gut verträglich.

\section{- Kommentar von Irina Falkenberg, Marburg}

\section{Lichttherapie im Winter wirkt auch bei nicht saisonaler Depression}

Es handelt sich um die erste randomisierte, kontrollierte Vergleichsstudie zur Effektivität von Lichttherapie und Pharmakotherapie bei der Behandlung der nicht saisonalen unipolaren Depression. Lichttherapie alleine oder in Kombination mit SSRI erwies sich als gut wirksam und gut verträglich. Angesichts der trotz existierender Pharmako- und psychotherapeutischer Behandlungsoptionen unbefriedigenden Remissionsraten bei der Depression sind die Studienergebnisse vielversprechend und erweitern die bisherige Rolle der Lichttherapie jenseits der saisonalen Depression. Überraschend ist das schlechte Abschneiden von Fluoxetin gegenüber Placebo. Die Breitengrade der Studienzentren sind mit Lichtverhältnissen im Winter in Mitteleuropa vergleichbar. Die Gruppengrößen in den einzelnen Armen waren recht klein, sodass Replikationen wichtig sind. Unklar bleibt, wie gut die Placebolichttherapie als Placebo erkannt wurde. Auch wurde die Studie während der dunk- len Jahreszeit - zwischen Oktober und März - durchgeführt. Daher ist zu erwarten, dass sich während der Frühjahrs- und Sommermonate die günstige Zusatzwirkung der Lichttherapie abschwächt, insbesondere wenn sich Patienten im Freien aufhalten.

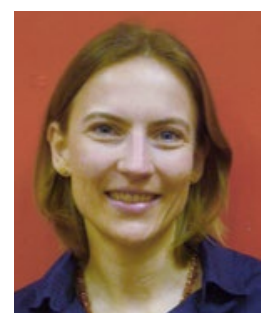

Dr. med. Irina Falkenberg, Marburg

Klinik für Psychiatrie und Psychotherapie Philipps-Universität Marburg - UKGMz E-Mail: irina.falkenberg@med.uni-marburg.de 